\title{
Prevention, Early Detection, and Reversal of Type-2 Diabetes using Collective System Design
}

\author{
Joseph J. Smith ${ }^{1, \star}$, Shahab A. Shah ${ }^{1}$, and David S. Cochran ${ }^{1}$ \\ ${ }^{1}$ Purdue University Fort Wayne, Fort Wayne, IN 46835 United States
}

\begin{abstract}
Type-2 Diabetes remains a progressive chronic disease that takes more and more lives every year. Small studies are beginning to show that a lifestyle approach could reverse type-2 diabetes in most individuals The key discoveries from these studies are captured and formulated into a System Design Map for Prevention, Early Detection, and Reversal of Type-2 Diabetes using Collective System Design. Collective System Design describes systems as four aspects that interrelate: tone, thinking, structure and work. The tone in Collective System Design fosters an environment that views human failure as a failure of the system design itself. The thinking in Collective System Design is communicated by Axiomatic Design (AD). With the Collective System Design Approach, a model for prevention, early detection, and reversal of chronic disease is developed. The motivation for this paper is the ability to save millions of lives through a sustainable lifestyle program by identifying the required diet and exercise.
\end{abstract}

\section{Introduction}

The approach for diabetes prevention and reversal presented in this paper is in reference to only type- 2 diabetes. Type-2 diabetes is commonly referred to as non-insulindependent diabetes, whereas type- 1 diabetes is characterized by the dependence on insulin [1]. However, to begin to understand how diabetes develops, an understanding of the role of insulin and the concept of resistance is essential. These concepts will be discussed in the literature review.

This paper explores the current hypothesis regarding the development of diabetes. The hypothesis, and the corresponding research, provides the foundation for the development of the Collective System Design (CSD) Map for Prevention, Early Detection, and Reversal of Type-2 Diabetes. An overview of CSD is provided in order to support the idea that the CSD Methodology is applicable to creating a model for early detection and reversal of chronic disease.

For the role of reversing existing cases of diabetes, a Very Low Carbohydrate Ketogenic Diet (VLCKD) is proposed to be the underlying treatment method[2,3]. The System Design Map presented in this paper expands upon the VLCKD to provide a systems engineering view of disease prevention and reversal. Systems Engineering at its core provides a sound foundation for developing disease prevention and reversal protocols, and this claim will be explained further in the methodology section of this paper.

First, the effectiveness of the VLCKD will be discussed by analyzing the supporting research. This paper does not attempt to argue that this approach is the only approach, or the best approach, it only provides standard

\footnotetext{
^e-mail: smitjj09@students.ipfw.edu
}

work for diabetes prevention, early detection, and reversal. This standard work provides a starting point for a continuous improvement process to further progress this approach by new research and discoveries.

\section{Literature Review}

The theory for the treatment of type- 2 diabetes presented within this paper is becoming prevalent in the medical literature [2-5]. The approach is comprised of using carbohydrate restriction to control insulin secretion with the underlying theory that insulin resistance is a response to chronic hyperglycemia and hyperinsulinemia $[1,4,6]$.

\subsection{The Development of Type-2 Diabetes}

Insulin, commonly referred to as the "fat storage hormone," is responsible for shuttling sugar out of the blood stream and into the cells $[1,4,5]$. When dietary carbohydrates from highly processed sources are ingested on a regular basis, large amounts of insulin must be circulated throughout the blood stream to keep glucose levels in a safe range. Over time, cells become resistant to the effects of insulin, thus, requiring more insulin to properly regulate glucose levels [4].

The pancreas, the organ responsible for the production of insulin, will continue to produce more and more insulin to overcome the resistance. After five to ten years, the pancreas will no longer be able to produce enough insulin to maintain blood sugar levels. This lack of insulin, or lack of effectiveness, occurs when an individual starts showing signs of high blood sugar, but the disease, and damage, have been progressing for many years [4]. 


\subsection{Reversing Type-2 Diabetes}

To reverse or eliminate insulin resistance, the stimulus must be removed. Being that insulin's role is to shuttle sugar out of the blood, removing that sugar in the first place, would reduce the need for insulin (i.e., remove the stimulus). All carbohydrates, including everything from unprocessed fruit to highly refined dessert items, are digested and broken broken down into sugar in the blood stream which stimulates the need for insulin [4].

Developing a resistance to insulin is synonymous with developing a resistance to alcohol. Over many years, as an individual consumes alcohol, he/she will become more resistant to its effects (i.e., requiring more alcohol to become intoxicated)[7]. To remove this resistance, one must abstain from alcohol. Similarly, with insulin resistance, one must abstain from the key drivers of insulin secretion, which are dietary carbohydrates, in order to reverse the resistance.

The result of restricting carbohydrates can be defined physiologically by recognizing the shift in the body's fuel source from one of glucose (sugar) to one of ketones (fat) [3]. This shift in homeostasis is commonly defined as nutritional ketosis and represents a range in blood ketone levels from 0.5 to $5 \mathrm{mM}$ [3]. The human body responds well to the lack of dietary carbohydrates by switching to fat for its primary fuel. Since fat is efficiently used as the main energy source, blood sugar levels are kept stable and at a low level. The small amount of glucose, required by the brain and other areas, is produced by the body through gluconeogenesis [3]. In this process, blood glucose is produced from glycerol: the backbone of triglycerides(or fat molecules).

The relevant literature on the use of a VLCKD to reverse diabetes shows great promise [2, 8-11]. One study in particular guides the motivation and design set forth in this paper [2]. The study, conducted by many of the leading physicians and researchers in the field of nutritional ketosis, set out to examine the effect of nutritional ketosis on patients with type- 2 diabetes. The study consisted of 262 participants. The program participants "were enrolled in an outpatient protocol providing intensive nutrition and behavioral counseling, digital coaching and education platform, and physician-guided medication management" [2]. At baseline, only $19.8 \%$ of the participants had a glycated hemoglobin percentage (HbA1c, a 3-month average of blood glucose levels) of less than $6.5 \%$ (the lower end of the diabetic range). After 10 weeks, $56.1 \%$ of participants had an A1c below 6.5\%.

\subsection{Early Detection of Type-2 Diabetes}

In addition to the research that supports the reversal of diabetes, the literature also examines potential blood markers that could predict diabetes at an earlier stage. Fig. 1 shows a representation of when the onset of diabetes occurs in reference to when the actual diagnosis takes place. The graph was adapted to depict how a person might feel during this time frame. This addition helps show why diabetes

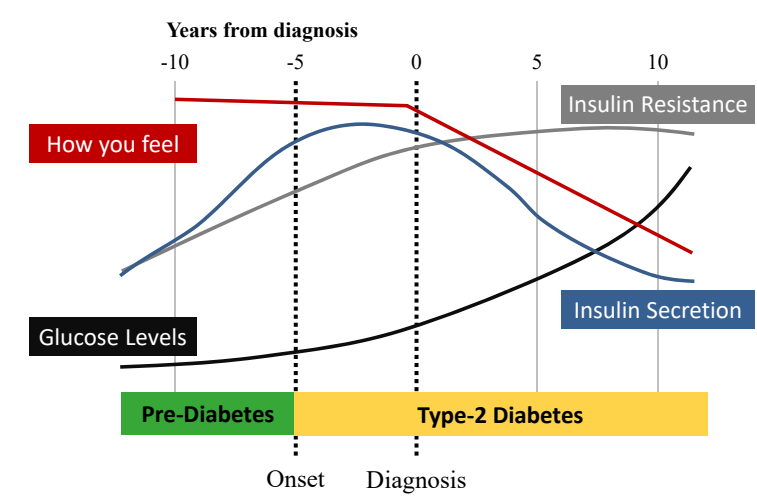

Figure 1. Time line depicting physiological changes 5 years before and after diagnosis. Adapted from [13].

is often detected around five years after the onset has taken place.

Recognizing that many physiological changes occur during the five year period prior to being diagnosed, provides insight into what tests could detect diabetes early on. Waiting for an individual to begin to feel worse allows for this five year period of physiological damage to go unnoticed, making further treatment and reversal even more difficult.

As ever increasing insulin levels are required to keep blood sugar within a normal range, an individual will not feel the biological effects that are taking place. Only after the pancreas can no longer produce enough insulin will a person begin to feel the effects of the resulting high blood sugar. Understanding this concept sheds light on what tests could predict diabetes earlier.

To further detail the possibility of early detection of diabetes, the Whitehall II study provides useful insight. In the Whitehall II study, 6,538 non-diabetic individuals were tested for fasting and two hour post-load glucose, and HOmeostasis Model Assessment (HOMA) for insulin sensitivity. After seven to nine years, 505 of these individuals were diagnosed diabetic. Retrospective trajectories of the above blood markers revealed that elevated fasting and two hour post-load glucose levels were present three years prior to diagnosis, and a steep decrease in HOMA insulin sensitivity began five years prior to diagnosis [12].

This research provides a foundation for which a system design map can be constructed. In the following section, the methodology and process behind constructing a system design map is discussed.

\section{Methodology}

The systems engineering methodology used for this research is known as Collective System Design (CSD). CSD is a product of Dr. David S. Cochran's work that began in the mid 2000's [14]. Systems Engineering is a discipline of engineering that focuses on the development and sustainment of systems to meet customer needs in a specific (path-dependent) manner throughout their life-cycle [15]. CSD is a methodical approach to product or system 


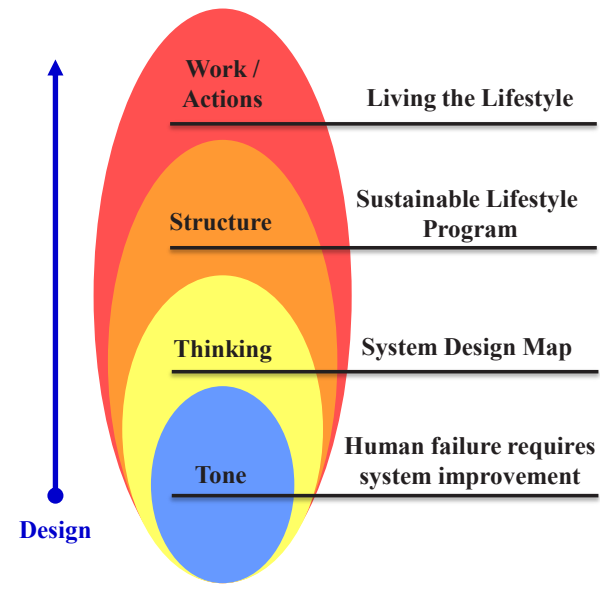

Figure 2. Flame Model of CSD for Prevention, Early Detection, and Reversal of Type-2 Diabetes. Adapted from [20].

design that facilitates a collective agreement regarding the requirements of the system or product [16-19].

The Flame Model of CSD in Fig. 2 represents the elements that exist within all systems, like the different layers within a flame. All elements are present within a system at any given time, and the outcome of each layer is either formed intentionally, or becomes unintentional due to the lack of attention given to that element. The work/actions and structure are the physical elements that we can see, and the thinking and tone are the hidden elements [20,21].

The "tone" of a system, or organization, represents the attitude and mindset of the people [22]. A proper tone facilitates a respectful environment that allows everyone to be engaged, and the understanding that a system should not let someone fail. When, not if, a failure occurs, the system and the people within the system should view the failure as an opportunity for improvement.

The Collective System Design Methodology starts with the tone, and the thinking is biased by the tone that is defined. Axiomatic Design is then used to codify the thinking layer of the Flame Model that results from the tone [22]. The tone should recognize that understanding customer needs is important to the success of the project. This recognition of customer needs is fundamental to the Axiomatic Design Theory [23].

Functional Requirements (FRs), or what the system must achieve, are derived from the customer needs. Physical Solutions (PSs) are then collectively chosen by the leadership team to achieve those requirements. A physical solution is a prerogative of the system and is referred to as Design Parameters (DPs) in Axiomatic Design [22, 23].

The thinking layer of the CSD Flame model seeks to understand the "why" before choosing the "how." Understanding the "why" is fundamental to the success of a system and has been recognized as a reason for failure in various types of industries even agriculture [24].

To understand when an FR is achieved, a measure (denoted by FRm) is placed on the FR. Likewise, a measure can be placed on a PS to identify when the PS is implemented correctly. These measures play a crucial role in continuously improving the system design by recognizing when FRs are not achieved.

\section{Collective System Design Map}

The legend in Fig. 3 provides the nomenclature for understanding the System Design Map for Prevention, Early Detection, and Reversal of Type-2 Diabetes. The left to right precedence within the top level of the system design map (see Fig. 5) is realized by understanding that the design is partially-coupled, or said another way, the design is path-dependent, meaning that there is an implementation sequence of the Physical Solutions. Fig. 4 shows an overview of the Collective System Design Map for Prevention, Early Detection, and Reversal of Type-2 Diabetes. This research began as a project for a Systems Engineering Course (SE530) taught by Professor David S. Cochran at Purdue University Fort Wayne. Later this research was expanded upon for the 2018 Purdue University Fort Wayne Student Research and Creative Endeavor Symposium [15]. In addition, this paper expands the Creative Endeavor Symposium research to include a prevention branch.

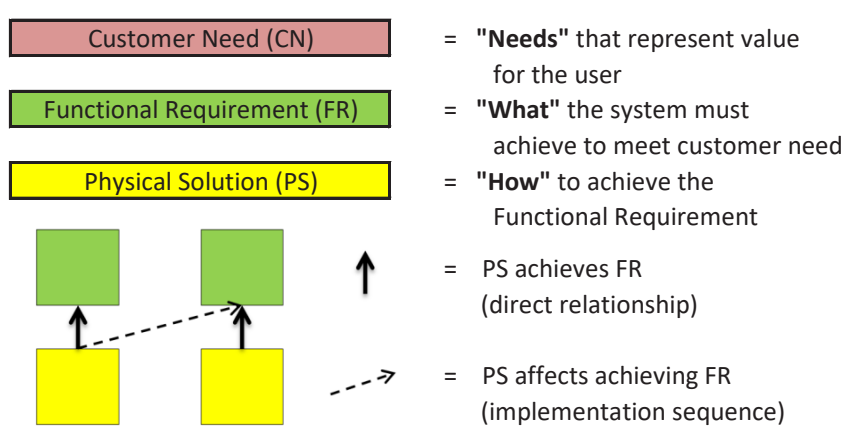

Figure 3. Legend regarding the Collective System Design Map

\subsection{Application of Axiomatic Design}

Axiomatic Design provides a scientific approach to design which can prevent designers from "tinkering" and ultimately reduce the amount of rework required. For the application of AD presented here, the prime focus was on the use of the Independence Axiom (Design Axiom 1). The Independence Axiom requires the designer to maintain the independence of the Functional Requirements [23]. Maintaining this independence will result in a specified implementation sequence of the Physical Solutions.

This implementation sequence is presented at a high level in Fig. 5. Thus, the design matrix for the second level (in Fig. 5) is decoupled and shows the dependencies among the PSs and FRs. Therefore, the implementation sequence of the different solutions is from left to right.

$$
\left(\begin{array}{l}
F R 11 \\
F R 12 \\
F R 13 \\
F R 14 \\
F R 15
\end{array}\right)=\left[\begin{array}{lllll}
X & 0 & 0 & 0 & 0 \\
X & X & 0 & 0 & 0 \\
X & 0 & X & 0 & 0 \\
X & 0 & X & X & 0 \\
X & 0 & X & X & X
\end{array}\right] \times\left(\begin{array}{c}
P S 11 \\
P S 12 \\
P S 13 \\
P S 14 \\
P S 15
\end{array}\right)
$$




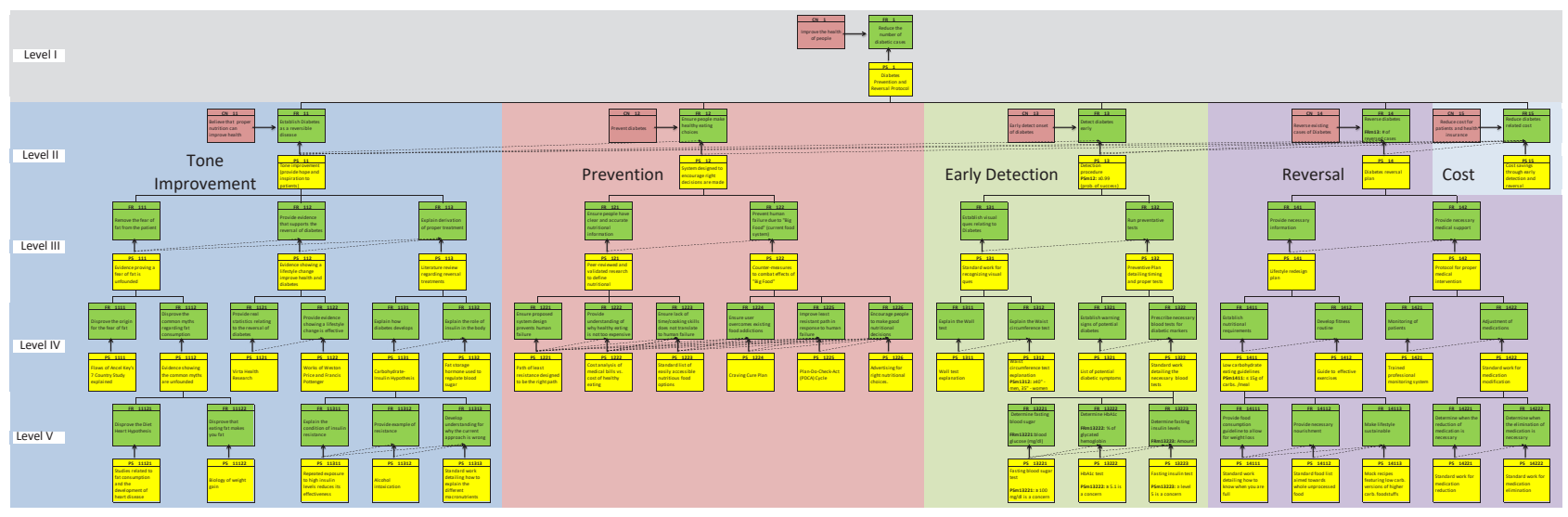

Figure 4. Overview of CSD Map for Prevention, Early Detection, and Reversal of Type-2 Diabetes (enlarge digital copy for full detail)

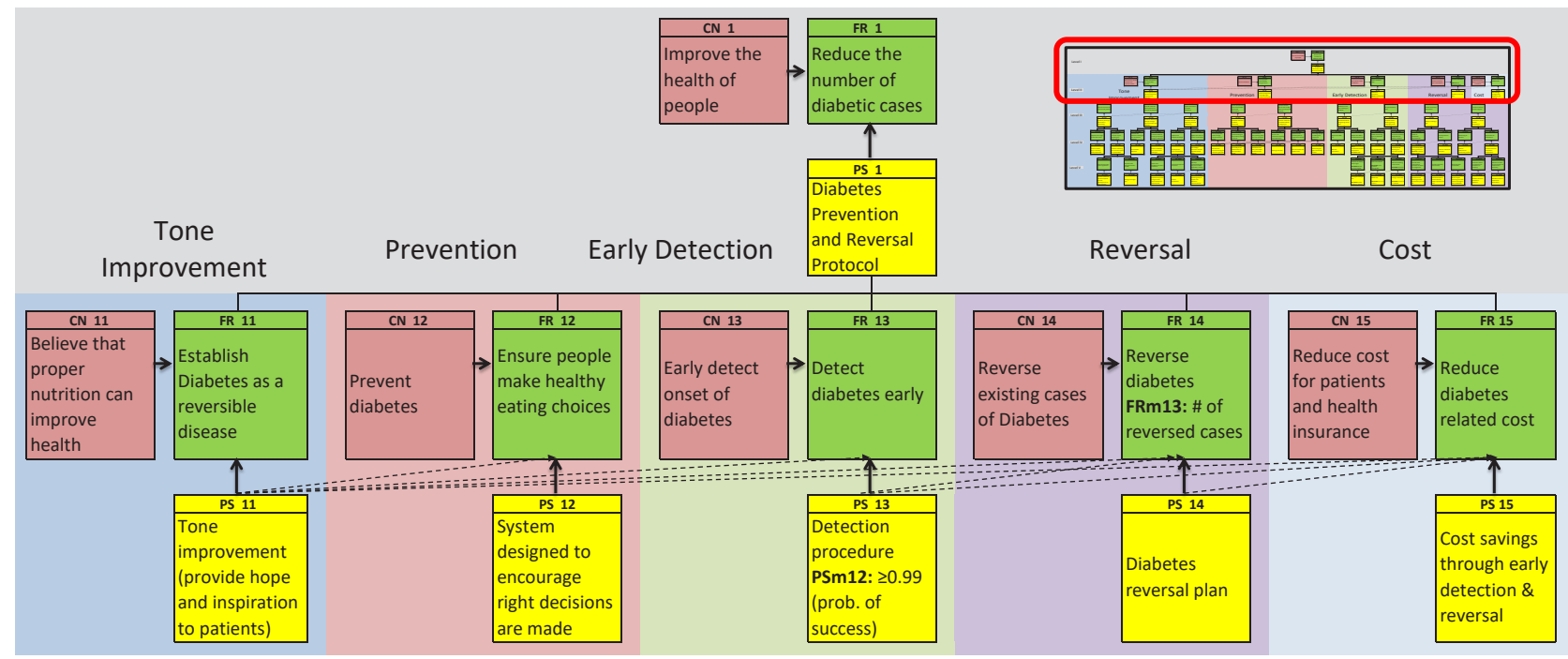

Figure 5. Top level of the Collective System Design Map for Diabetes Reversal

\section{Top-Level}

The top-level FR for the CSD map (FR1) is to "Reduce the number of diabetic cases," and the proposed PS to achieve this FR (PS1) is the "Diabetes Prevention and Reversal Protocol." This protocol represents an amalgamating PS that is further decomposed into additional FRs, which include tone improvement, prevention, early detection, reversal, and cost. The details of each branch are explained in the following sections.

\subsection{Tone Improvement}

Tone improvement, in the case of the CSD map for diabetes reversal (see Fig. 6), helps provide hope to everyone involved through education. This hope comes from understanding that being diabetic is not a death sentence or a predefined destiny [1]. The potential to reverse existing cases or prevent future ones, lies in the design of this CSD map for diabetes reversal and the openness of people to accept a paradigm shift in treatment.

A lifestyle program that removes carbohydrates from a person's diet must replace those calories with fat. For the last century, fat has been viewed as a key player in the development of heart related diseases and obesity, but there may be more to the story [3, 5, 27-29]. Asking patients to remove carbohydrates (i.e, potatoes, rice, whole grains, sugar, etc...) and to replace those calories with fat (i.e., butter, bacon, steak, cheese, avocados, etc...) will require educating them on why such a diet will provide wellness. In addition, explaining the biological mechanism behind the chosen treatment method (PS1131) will further provide confidence in accepting the VLCKD as a treatment method.

When the tone of people is that systems fail and people do not, and diabetes is not a death sentence, an environment for reversal is established. This proper tone of the people involved in the system represented by the CSD map for diabetes reversal is first and foremost to reducing the number of diabetic cases. Without the recognition that people have control over their health, patients will lack the courage to engage in the prevention, early detection, and reversal process. Providing the necessary information for the patients to understand why this treatment method is not only safe, but also effective, is paramount to the success of 


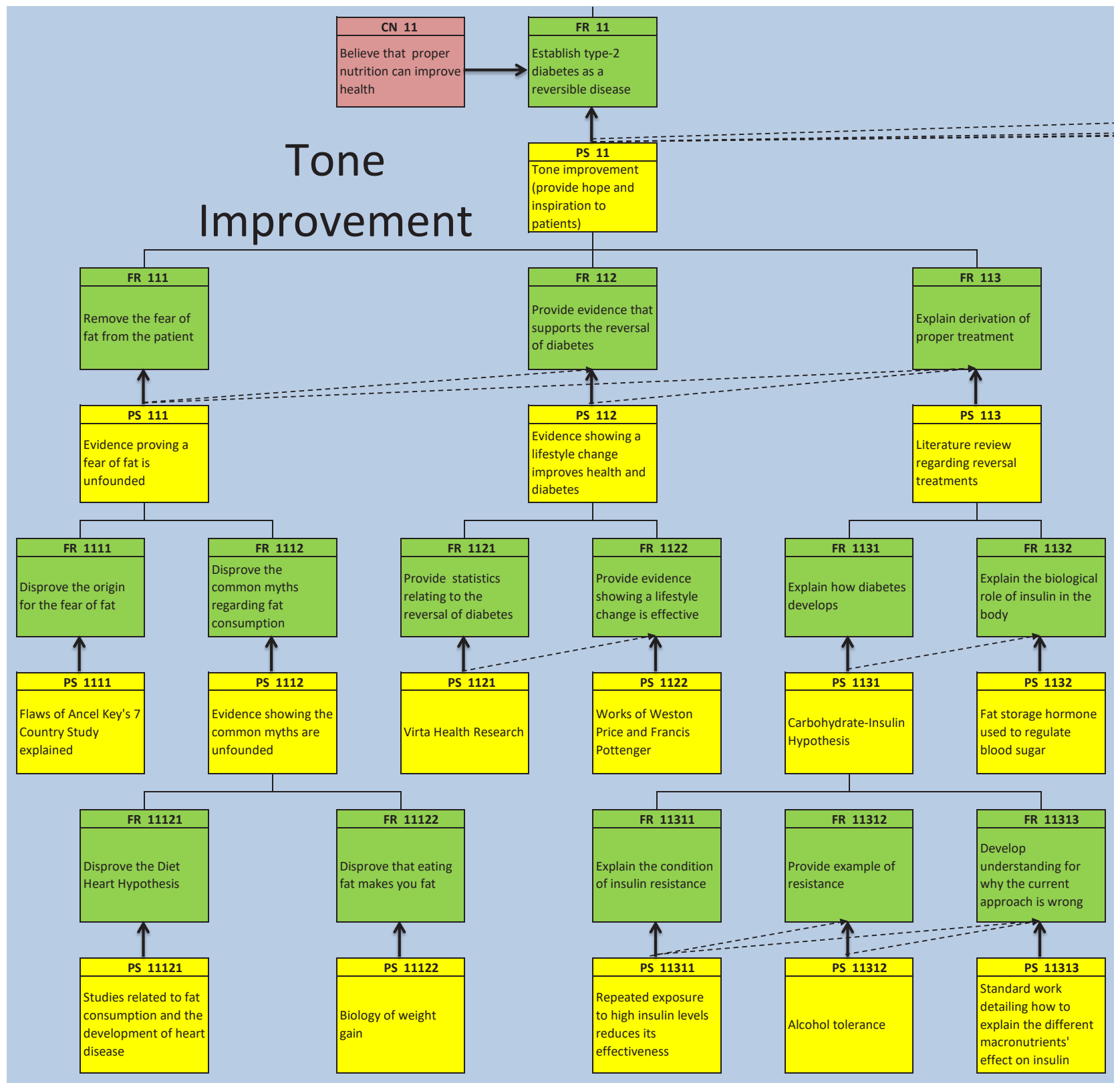

Figure 6. Tone Improvement Branch of the CSD Map for Diabetes Reversal; [2] supports PS1121, and [25, 26] supports PS1122.

reversing diabetes. Dr. Jason Fung presents much of this information to his patients to explain why the long standing treatment for type-2 diabetes, to inject insulin, should not be the standard for treating type-2 diabetes [4].

The path-dependency of the CSD map illustrates that the ability to educate and provide hope and encouragement (Tone Improvement) will affect a system's ability to prevent, early detect, and reverse diabetes. With this understanding, the Prevention branch is next in the implementation sequence.

\subsection{Prevention}

The FR to prevent future cases of diabetes is to "ensure people make healthy eating choices" (FR12), and this FR is achieved by a "system designed to encourage making the right nutritional decisions" (PS12) (see Fig. 7). For an individual to make the right decision, one has to know what the right decision is, but, in many cases, this information alone will not be enough to drive change. For example, food addictions and other habits will need to be overcome to ensure the right eating choices are made. In the Toyota Production System, defects were prevented by mistake-proofing operations (Poka-yoke) [31]. Viewing wrong lifestyle choices as a type of defect allows for mistake-proofing various situations that patients will encounter. For example, having easy access to healthy food will help prevent making wrong food choices in times of stress (PS12223).

The current food supply is one that has created a society of overfed but undernourished people [27, 29]. Both Gary Taubes and Nina Teicholz, investigative journalists, have presented the politics and unfortunate events that 


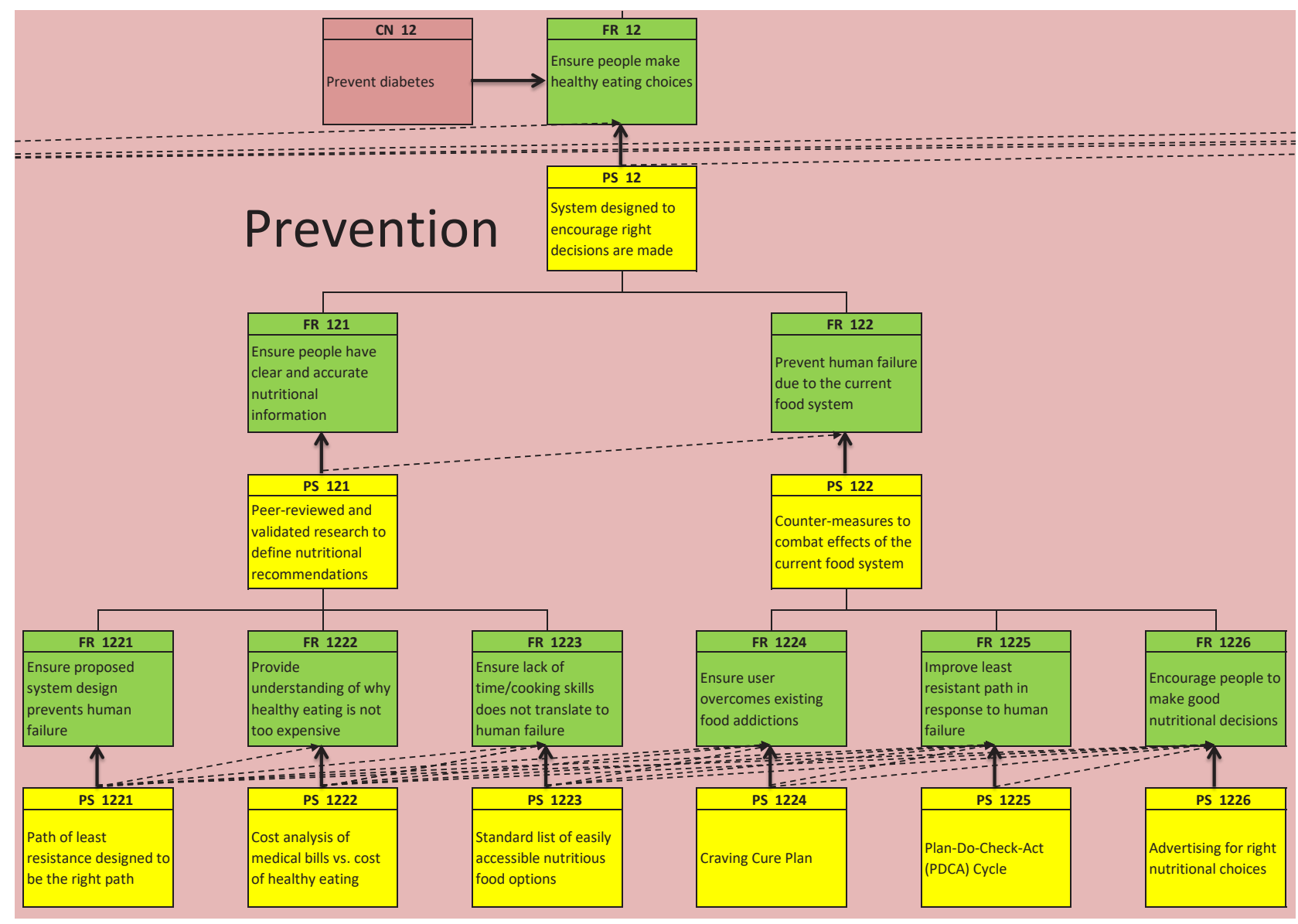

Figure 7. CSD Map for Diabetes Reversal; [30] supports PS1224.

have led to the dietary recommendations over the last century. Arguing whether the dietary recommendations where based on the best known science at the time, or on political or financial influence, is not the intent of this paper, but it is important to recognize that the current food system is failing to improve the health of society. Every indicator of health is moving in the wrong direction and people are not to blame.

Understanding that the current food supply is not designed for wellness allows for a new system to be designed to prevent failure in response to the current food supply. The Prevention branch of the CSD Map for Prevention, Early Detection, and Reversal of Type-2 Diabetes is built on this understanding. Understanding how to prevent diabetes, leads to the ability to early detect future cases.

\subsection{Early Detection}

The motivation for the Early Detection branch (see Fig. 8) is represented in Fig. 1. Recognizing that physiological changes begin to occur around five years prior to diagnosis provides a large window to begin or to improve prevention measures. Health markers involving blood glucose levels will not detect diabetes as early as those relating to insulin. Blood glucose measures, including fasting glucose and $\mathrm{HbA} 1 \mathrm{c}$ are the standard for predicting and diagnosing diabetes [4].
Measuring blood glucose is analogous to measuring the water that spills out of a bucket once it is full, but measuring insulin would be like measuring the height of the water in the bucket. If the bucket spilling over represents the diagnosis of diabetes, it is clear that measuring the height of the water (insulin) would predict the overflow long before it occurs.

Understanding the difference in measuring insulin versus blood glucose leads to a detection procedure comprised of visual cues (PS131), fasting blood sugar (PS13221), HbA1c (PS13222), and fasting insulin (PS13223). With this detection procedure, future diabetic cases can be predicted early on, or currently undiagnosed diabetics can be properly diagnosed. The ability of the system represented by the CSD map for diabetes reversal to provide early detection and proper diagnosis affects the ability to reverse existing cases of diabetes.

\subsection{Reversal}

The Diabetes Reversal Plan (PS14) referred to in Fig. 9 is built on the theory presented in the literature review of this paper. The approach is comprised of using carbohydrate restriction to control insulin secretion with the underlying theory that insulin resistance is a response to chronic hyperglycemia and hyperinsulinemia $[1,4,6]$. 


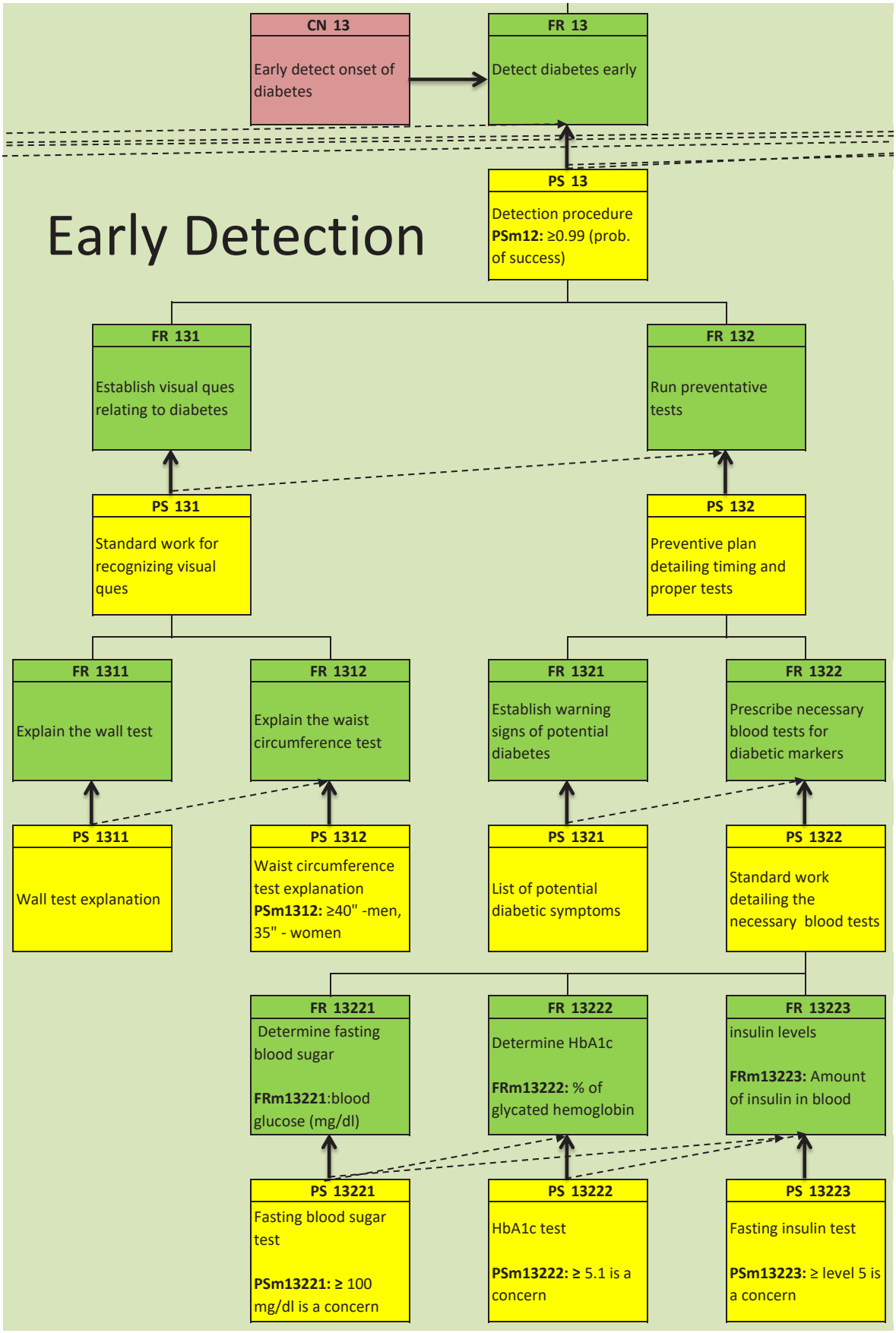

Figure 8. Early Detection Branch of the CSD Map for Diabetes Reversal

Continuing with the bucket analogy, if water represents blood sugar, then reversing and preventing diabetes is based on removing that sugar (i.e., reduce the amount of water in the bucket). Carbohydrates, even in naturally occurring fruits and vegetables, break down to blood glucose [3]. Thus, these food items represent adding water to the bucket.

Carbohydrate restriction was first documented in 1864 in William Banting's Letter on Corpulence as a diet for weight loss [32], but more recently it has been viewed as a "fad" diet because it restricts natural food products such as potatoes, rice, and fruit. Although the intent of this paper is not to argue which diet approach is most effective, the validity of the low carbohydrate diet is an important point to establish in order to understand why it was used as the basis for this system design.

Processed sugar and white flour are food products that are highly refined from their natural state. Refining these food items in order to incorporate them into many processed food products increases the absorption rate of glucose into the blood stream. Consuming these highly processed foodstuffs is unnatural and would not be possible without modern processing capabilities[25]. Recognizing that processed (unnatural) food can harm the body and put it into a diseased state provides an argument for an unnat- 


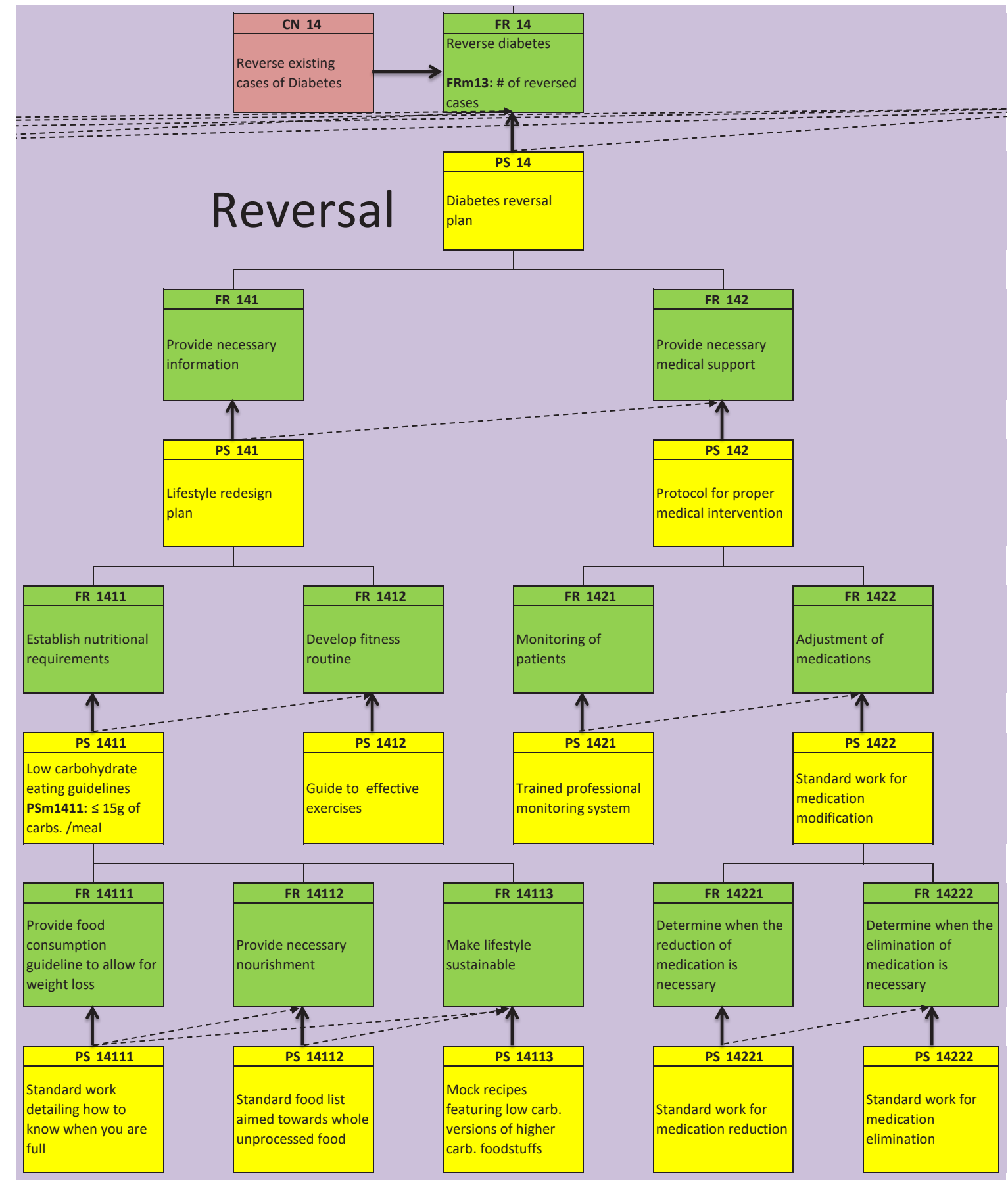

Figure 9. Reversal Branch of the System Design Map

ural treatment method (or what many people refer to as a fad diet.)

The Reversal branch of the CSD map depicts the use of a low carbohydrate diet to reverse diabetes in addition to reducing and eliminating any medication the patient may be taking. This support, in terms of medication reduction and elimination, is a key aspect to maintaining the safety and health of the patient. Standard work for medication management can provide a baseline for current practice, but it ultimately establishes a standard that can continuously be improved. The process for continuous improvement is explained in the following section.

\section{Removing Blame by Continuous Improvement}

To understand the applicability of applying a system design methodology to disease management, an example from a manufacturing setting may best set the stage for this argument. Within manufacturing, there is a never ending pursuit for perfect quality. One aspect of achieving this requirement is to ensure that operator human errors do not translate to defects (FR-Q113 from the Manufacturing System Design Decomposition (MSDD))[33]. To best achieve this requirement, the solution is to mistake proof the operations (Poka-Yoke)(PS-Q113). Therefore, 
an operator should not be able to install something backwards, or forget to install something. When a failure does occur due to an operator, a well designed system will look to improve the system and prevent further occurrences of this failure mode.

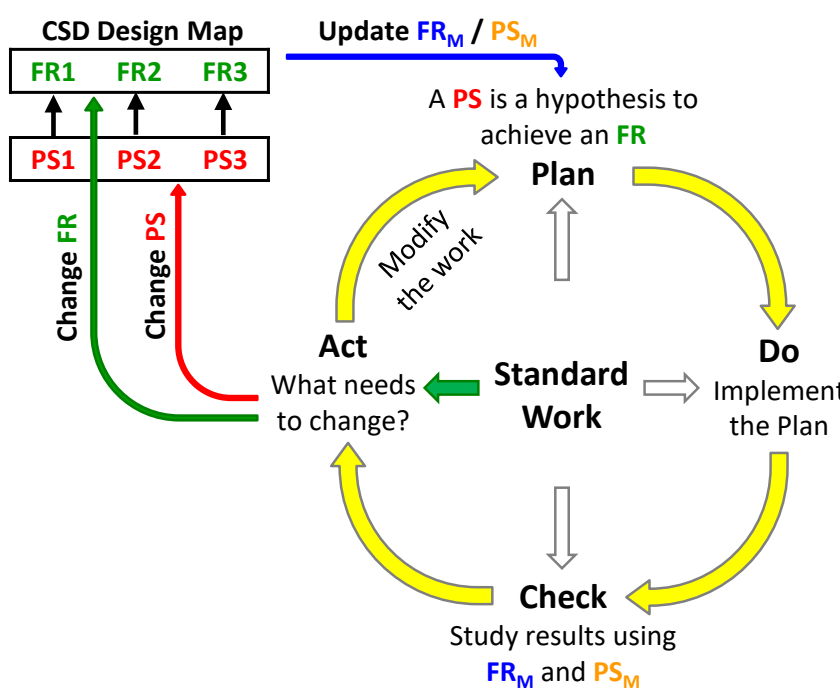

Figure 10. Plan-Do-Check-Act (PDCA) Cycle [34]

In the case of type- 2 diabetes, the PSs of the Collective System Design Map are implemented through standard work. Thus, when a chosen PS does not achieve an FR, a continuous improvement process modifies the map to reduce and account for human failure. The Collective System Design Methodology provides a structured approach to the process of continually improving the system design map. This approach, known as the Plan-Do-Check-Act (PDCA) cycle, has been adapted from Dr. Deming's work to provide a process for adapting a system design map[35]. The PDCA cycle presented in Fig. 10 provides 4 ways to modify a system design map. The 4 possible ways to "act" include modifying the work, modifying the physical solutions, modifying the functional requirement, or modifying the measures on the FR or PS.

With this systems view of disease prevention and reversal, an individuals will power, or the lack of, can no longer be cited as the cause of poor results. When an individual is overcoming food addictions, which will often be the case, binging on carbohydrates does not represent a lack of character, it represents an opportunity to improve the system. The systems engineer has the responsibility of improving a system in response to human failure [36]. For this reason, a systems engineering view provides the core mindset required to help overcome food and lifestyle habits.

\section{Conclusion}

With the growing prevalence of type- 2 diabetes around the world, a new approach needs to be taken for preventing and reversing this chronic disease. The current system "design," as it has evolved, rewards behaviors that are not aligned with the CSD Map presented here. For instance, if the lifestyle re-design plan from PS141 is implemented, the pharmaceutical treatments may not be required.

A new system, defined by a Collective System Design map for the Prevention, Early Detection, and Reversal of Type-2 Diabetes provides a way to document the best-known practices right now, in addition to providing a way to continually improve the map as more research is completed. This product of Axiomatic Design provides a road map to implement such a system. By understanding the path-dependency of the design prior to implementation, rework is minimized and the design can be clearly communicated to the individuals involved with implementation.

The understanding of the path-dependency of a system to reverse diabetes provided the realization that tone improvement would affect the rest of the system design. Patients' would require support and a practical understanding of the treatment in order to be successful. Thus, the outcome of implementing the PSs within the tone improvement branch will provide this support and practical understanding.

With the Axiomatic Design approach to disease management, the system design map proposed in this paper can not only be replicated across the world by different clinics, but this map can also be used as a model for the management of other chronic diseases. With this systems thinking view of disease management, failure is viewed as an opportunity to improve the system, which stands in stark contrast to blaming a patient for their lack of will power.

\section{References}

[1] R.K. Bernstein, Dr. Bernstein's Diabetes Solution, 4th edn. (Hachette Book Group, 2011)

[2] A.L. McKenzie, S.J. Hallberg, B.C. Creighton, B.M. Volk, T.M. Link, M.K. Abner, R.M. Glon, J.P. McCarter, J.S. Volek, S.D. Phinney, JMIR Diabetes 2, e5 (2017)

[3] S.J. Volek, D.S. Phinney, The Art and Science of Low Carbohydrate Living (Beyond Obesity, LLC, 2011)

[4] J. Fung, The Diabetes Code: Prevent and Reverse Type 2 Diabetes Naturally, 1st edn. (Greystone Books, 2018)

[5] G. Taubes, The Case Against Sugar, 1st edn. (Alfred A. Knopf, 2016)

[6] R.D. Feinman, J.S. Volek, Scandinavian Cardiovascular Journal 42, 256 (2008)

[7] Alcohol and Tolerance - Alcohol Alert No. 281995 (1995), https://pubs.niaaa.nih.gov/ publications/aa28.htm

[8] S.J. Hallberg, A.L. McKenzie, P.T. Williams, N.H. Bhanpuri, A.L. Peters, W.W. Campbell, T.L. Hazbun, B.M. Volk, J.P. McCarter, S.D. Phinney et al., Diabetes Therapy 9, 583 (2018)

[9] R. Tate, The Magic Pill (2018)

[10] A. Accurso, R.K. Bernstein, A. Dahlqvist, B. Draznin, R.D. Feinman, E.J. Fine, A. Gleed, 
D.B. Jacobs, G. Larson, R.H. Lustig et al., Nutrition \& Metabolism 5, 9 (2008)

[11] L.R. Saslow, A.E. Mason, S. Kim, V. Goldman, R. Ploutz-Snyder, H. Bayandorian, J. Daubenmier, F.M. Hecht, J.T. Moskowitz, Journal of Medical Internet Research 19, e36 (2017)

[12] A.G. Tabák, M. Jokela, T.N. Akbaraly, E.J. Brunner, M. Kivimäki, D.R. Witte, The Lancet 373, 2215 (2009)

[13] American Diabetes Advocates (ADA) - National Diabetes Information Clearinghouse Home, http://www.diabetesadvocates.info/ Complications.html

[14] D.S. Cochran, Journal of Cost Management 20, 20 (2006)

[15] J. Smith, S. Shah, Disease Management Through Collective System Design (CSD) Approach (2018), first place recipient, https://opus.ipfw.edu/ stu_symp2018/47

[16] D.S. Cochran, J. Oxtoby, M. Hensley, J. Barnes, Journal of Enterprise Transformation 7 (2017)

[17] D.S. Cochran, The Journal of Reliability, Maintainability, Supportability in Systems Engineering Winter Journal, 6 (2009)

[18] D.S. Cochran, The Journal of Reliability, Maintainability, Supportability in Systems Engineering Spring Journal, 16 (2010)

[19] D.S. Cochran, in Lean Accounting, edited by J. Stenzel (John Wiley and Sons, New Jersey, 2007)

[20] D.S. Cochran, J. Swartz, Sustaining Improvement through Tone in Collective System Design (Anaheim, CA, 2016)

[21] D.S. Cochran, Systems Approach to Sustain Lean Organizations, in SAE International World Congress (Cobo Hall Detroit, MI, 2007), Vol. 2007-01-1377
[22] D.S. Cochran, Collective System Design in Industrial and Systems Engineering Education (Louisville, KY, 2010)

[23] N.P. Suh, Complexity: Theory and Applications (Oxford University Press, 2005)

[24] J. Salatin, You Can Farm, 1st edn. (Polyface, 1998)

[25] W. Price, Nutrition and Physical Degeneration: A Comparison of Primitive and Modern Diets and Their Effects, 8th edn. (Price Pottenger Nutrition, 1939)

[26] Pottenger's Cats: A Study in Nutrition, 2nd edn. (Price Pottenger Nutrition, 2012)

[27] N. Teicholz, The Big Fat Surprise: Why Butter, Meat and Cheese Belong in a Healthy Diet, 1st edn. (Simon \& Schuster, 2014)

[28] M. Pollan, The Omnivore's Dilemma, 1st edn. (The Penguin Press, New York, 2006)

[29] G. Taubes, Good Calories, Bad Calories, 1st edn. (Alfred A. Knopf, 2007)

[30] J. Ross, The Craving Cure, 1st edn. (Flat Iron Books, 2017)

[31] S. Shingo, A. Dillon, A Study of the Toyota Production System: From an Industrial Engineering Viewpoint (Produce What Is Needed, When It's Needed), 1 st edn. (Productivity Press, 1989)

[32] W. Banting, Letter on corpulence : addressed to the public (New York, 1864)

[33] D.S. Cochran, J.T. Foley, B. Zhuming, International Journal of Production Research 55, 870 (2017)

[34] D.S. Cochran, J. Barnes, Sustainable Enterprise Design (Fort Wayne, IN, 2015)

[35] W. Deming, Out of the Crisis, 1st edn. (The MIT Press, 2000)

[36] D. Long, Reimagining Systems Engineering (2017) 